\title{
On Types of Solutions of the Second Order Nonlinear Boundary Value Problems
}

\author{
Maria Dobkevich, ${ }^{1}$ Felix Sadyrbaev, ${ }^{1}$ Nadezhda Sveikate, ${ }^{2}$ and Inara Yermachenko ${ }^{2}$ \\ ${ }^{1}$ Institute of Mathematics and Computer Science, Rainis Boulevard 29, Riga LV-1459, Latvia \\ ${ }^{2}$ Daugavpils University, Parades Street 1, Daugavpils LV-5400, Latvia
}

Correspondence should be addressed to Felix Sadyrbaev; felix@latnet.lv

Received 28 April 2014; Accepted 20 June 2014; Published 12 August 2014

Academic Editor: Tongxing Li

Copyright (c) 2014 Maria Dobkevich et al. This is an open access article distributed under the Creative Commons Attribution License, which permits unrestricted use, distribution, and reproduction in any medium, provided the original work is properly cited.

We review the results concerning types of solutions of boundary value problems for the second order nonlinear equation $\left(l_{2} x\right)(t)=$ $f\left(t, x, x^{\prime}\right)$, where $\left(l_{2} x\right)(t)$ is the second order linear differential form. The existence results and the multiplicity results are stated in terms of types of solutions.

\section{Introduction}

The aim of this paper is to gather the results concerning types of solutions of the second order nonlinear boundary value problem

$$
\begin{gathered}
x^{\prime \prime}=f\left(t, x, x^{\prime}\right), \\
a_{1} x(a)+a_{2} x^{\prime}(a)=A, \quad b_{1} x(b)+b_{2} x^{\prime}(b)=B
\end{gathered}
$$

and particular cases. A great portion of results is formulated to the Dirichlet boundary conditions:

$$
x(a)=A, \quad x(b)=B .
$$

A number of the below listed results concern the more general equation:

$$
\left(l_{2} x\right)(t)=f\left(t, x, x^{\prime}\right)
$$

where $\left(l_{2} x\right)(t):=x^{\prime \prime}+p(t) x^{\prime}+q(t) x$.

Vast literature is devoted to boundary value problems (BVP in short) of type (1), (3). The interested reader may consult the books [1-6]. The main issues are the existence, multiplicity, and properties of solutions.

Some existence results will be given in the next section. As to the uniqueness, we emphasize the result by Erbe [7] where the conditions for uniqueness of a solution to BVP (1), (3) are given in terms of nonoscillatory behavior of equations of variations for any solution of (1).

Properties of solutions of BVPs are numerous. Why are we interested exactly in types of solutions? What is the type of a solution?

The answer to these inquiries will be given later.

The paper is organized as follows. In the second section some existence results are provided and the notions of the upper and lower functions are introduced. The third section contains information on uniqueness of solutions. The results formulated in terms of equation of variations are selected. The fourth section contains brief account of the works by Knobloch, Jackson, Schrader, and Erbe dealing with the properties of solutions of BVP which can be expressed in terms of equations of variations. The fifth section deals mainly with solutions of BVP which cannot be approximated by monotone sequences of solutions. The respective equations of variations are oscillatory. The sixth section provides information on the Neumann BVP. In the seventh section connection between types of solutions and the multiplicity of solutions is discussed. In the eighth section quasilinear BVP are considered. Properties of the linear part of a quasilinear BVP influence both the properties of solutions of BVP and the number of solutions. The quasilinearization process is considered. In the ninth section the results concerning 
application of the quasilinearization process to resonant problems are reviewed. The last tenth section contains conclusions and final remarks.

\section{Existence}

The following result is very easy to understand when asking for conditions which ensure the existence of a solution to the problem (1), (3). By a solution it is meant a $C^{2}$-function $x(t)$ which satisfies both (1) in the interval $[a, b]$ and boundary conditions (3).

Theorem 1. The problem (1), (3) has a solution $x(t)$ if a function $f\left(t, x, x^{\prime}\right)$ is continuous and bounded $(|f|<M$ for some constant $M)$.

This theorem is known also as the Picard theorem.

The following result needs some definitions.

Definition 2. A function $\alpha \in C^{2}([a, b], R)$ is called a lower function for (1) if $\alpha^{\prime \prime}(t) \geq f\left(t, \alpha(t), \alpha^{\prime}(t)\right), \forall t \in[a, b]$.

Similarly, a function $\beta \in C^{2}([a, b], R)$ is called an upper function for (1) if $\beta^{\prime \prime}(t) \leq f\left(t, \beta(t), \beta^{\prime}(t)\right), \forall t \in[a, b]$.

To formulate the existence result we need also the Nagumo condition; there exists a positive valued continuous function $\varphi(y)$ such that

(1) $\left|f\left(t, x, x^{\prime}\right)\right| \leq \varphi\left(\left|x^{\prime}\right|\right), \forall\left(t, x, x^{\prime}\right) \in \omega(\alpha, \beta) \times R$, where $\omega(\alpha, \beta)=\{a \leq t \leq b, \alpha(t) \leq x \leq \beta(t)\}$

(2) $\int_{\lambda}^{N}(s d s / \varphi(s))>\max _{[a, b]} \beta(t)-\min _{[a, b]} \alpha(t)$.

The latter condition is fulfilled if the integral $\int_{0}^{+\infty}(s d s /$ $\varphi(s))=+\infty$. In particular, this is true if $\varphi(y)=c_{1}+c_{2} y^{2}$.

The next theorem provides generalization of Picard theorem. If $f$ is bounded in (1) then the lower and upper functions can be constructed in the form of quadratic parabolas.

Theorem 3. Let there exist lower and upper functions $\alpha(t)$ and $\beta(t)$ such that

(1) $\alpha(a) \leq A \leq \beta(a), \alpha(b) \leq B \leq \beta(b)$;

(2) $\alpha(t) \leq \beta(t), \forall t \in[a, b]$;

(3) the Nagumo condition holds with respect to a given pair $(\alpha, \beta)$.

Then there exists a solution $x(t)$ of the problem (1), (3) with the graph $(t, x(t))$ belonging to $\omega(\alpha, \beta)$.

The proof is by considering the modified function $F$ which coincides with $f$ for $(t, x) \in \omega(\alpha, \beta)$ and for $\left|x^{\prime}\right|<$ $N$, where $N$ is a constant appearing in the above Nagumo condition. The proof is not trivial, but it is not also too complicated and can be found in most of the above mentioned books on BVPs.

Remark 4. It is to be mentioned that there are multiple generalizations of definition of lower and upper functions. We recommend consulting the book $[8]$ and papers $[9,10]$.
Remark 5. There are also multiple generalizations of the Nagumo condition. We would like to mention the so-called one-sided Nagumo type conditions. The main idea is that, depending on the type of boundary conditions, the restrictions on $f$ in the form $f<\varphi$ or $f>-\varphi$ should be imposed only on parts of the set $\omega(\alpha, \beta)$. We recommend looking at [11] and some results in [3]. For relatively complete report on one-sided Nagumo type conditions one may consult the paper [12].

For the more general problem (1),

$$
\begin{aligned}
& a_{1} x(a)-a_{2} x^{\prime}(a)=A, \\
& b_{1} x(b)+b_{2} x^{\prime}(b)=B,
\end{aligned}
$$

where $A, B \in \mathbb{R}, a_{1}, b_{1} \in \mathbb{R}, a_{2}, b_{2} \in \mathbb{R}^{+}:=(0,+\infty), a_{1}^{2}+a_{2}^{2}>$ 0 and $b_{1}^{2}+b_{2}^{2}>0$; the following is true.

Theorem 6. Let the condition (1) in Theorem 3 be replaced with

$$
\begin{array}{ll}
a_{1} \alpha(a)-a_{2} \alpha^{\prime}(a) \leq A, & a_{1} \beta(a)-a_{2} \beta^{\prime}(a) \geq A, \\
b_{1} \alpha(b)+b_{2} \alpha^{\prime}(b) \leq B, & b_{1} \beta(b)+b_{2} \beta^{\prime}(b) \geq B .
\end{array}
$$

Then there exists a solution $x(t)$ of the problem (1), (5) with the graph $(t, x(t))$ belonging to $\omega(\alpha, \beta)$.

\section{Uniqueness}

Of multiple uniqueness results concerning the problem (1), (3), we mention the following theorem by Erbe [7].

First recall that, for any solution $x(t)$ of $(1)$, a linear equation of variations

$$
y^{\prime \prime}=f_{x}\left(t, x(t), x^{\prime}(t)\right) y+f_{x^{\prime}}\left(t, x(t), x^{\prime}(t)\right) y^{\prime}
$$

can be considered. It is used to say that the equation of variations is disconjugate in the interval $[a, b]$ if no nontrivial solution $y(t)$ of $(7)$ has two zeros in $[a, b]$, or, equivalently, a solution of the initial value problem (7) $y(a)=0, y^{\prime}(a)=1$ does not vanish in $(a, b]$.

Theorem 7 (Erbe). Suppose the Nagumo condition is fulfilled. The problem (1), (3) has at most one solution if for any solution $x(t)$ of (1) the respective equation of variations (7) is disconjugate in $[a, b]$.

Therefore, if there are multiple solutions of the BVP, some of equations of variations are not disconjugate in the interval.

\section{Knobloch-Jackson-Schrader-Erbe Results on $B$-Solutions}

It was Knobloch [13] who observed first that (1) in presence of regularly ordered $(\alpha \leq \beta)$ the upper $\beta$ and lower $\alpha$ functions have a specific solution $\xi(t)$ which possesses the property $(B)$ 
and are located in a region $\omega(\alpha, \beta)$. He wrote "We say that a solution $\xi$ " of (1) on a compact interval $I$ of the real line "has property $(B)$ if there exists a sequence of solutions $\xi_{n}$ such that

(i) $\xi_{n} \rightarrow \xi, \xi_{n}^{\prime} \rightarrow \xi^{\prime}$ uniformly on $I$;

(ii) $\xi-\xi_{n} \neq 0$ and has the same sign for all $n \geq 1$ and all $t \in I$

(iii) $\left|\xi_{n}^{\prime}-\xi^{\prime}\right| \leq c\left|\xi_{n}-\xi\right|$ for all $n \geq 1$ and all $t \in I$, where $c$ is a constant independent of $n$ and $t$.

Later Knobloch [14] formulated property $\left(B^{*}\right)$ : a solution $x$ of (1) is said to have property $\left(B^{*}\right)$ on $[a, b]$ in case there exists a sequence of solutions $x_{n}$ of (1), all having property $(B)$ on $[a, b]$, such that $x_{n} \rightarrow x$ and $x_{n}^{\prime} \rightarrow x^{\prime}$ uniformly on $[a, b]$.

It was proved in $[14$, Lemma 2.1] that if $\xi(t)$ is a solution of (1) having property $\left(B^{*}\right)$ with respect to $[0, T]$ and if $f, f_{x}$, and $f_{x^{\prime}}$ are continuous functions on some neighborhood of the curve $(t, \xi(t), \xi(t))$, $(0<t<T)$, then the equation of variations (7) is disconjugate on $(0, T)$.

In the work [14] the boundary value problem

$$
\begin{aligned}
x^{\prime \prime}=f(t, x, x), \quad x(0) & =\sigma(x(0)), \\
x(T) & =\tau(x(T)),
\end{aligned}
$$

where $\sigma, \tau$ are continuously differentiable functions, defined on the intervals $[\alpha(0), \beta(0)],[\alpha(T), \beta(T)]$, respectively and subject to the conditions

$$
\begin{array}{ll}
\alpha(0) \geq \sigma(\alpha(0)), & \beta(0) \leq \sigma(\beta(0)), \\
\alpha(T) \leq \sigma(\alpha(T)), & \beta(T) \geq \sigma(\beta(T)),
\end{array}
$$

was considered. It was proved in [14] that, provided the existence of a regular pair $(\alpha \leq \beta)$ of lower and upper functions $\alpha$ and $\beta$, satisfying (9), and under a mild Nagumo type condition, a solution $x(t)$ of the BVP (8) exists such that $x$ possesses the property $\left(B^{*}\right)$ and $\alpha \leq x \leq \beta$.

After the first paper by Knobloch [13] Jackson and Schrader [15] considered the Dirichlet problem (1), (3). They introduced the property $\left(B^{\prime}\right)$, which slightly differs from the property $\left(B^{*}\right)$ :

(i) $\xi_{n} \rightarrow \xi, \xi_{n}^{\prime} \rightarrow \xi^{\prime}$ uniformly on $I$;

(ii) $\xi-\xi_{n} \neq 0$ and has the same sign for all $n \geq 1$ and $a \leq t<b$, or for all $n \geq 1$ and $a<t \leq b$;

(iii) for each $0<\delta<(1 / 2)(b-a)$ there is a constant $c$ depending on $\delta$ but not on $n$ and $t$ such that $\left|\xi_{n}^{\prime}-\xi^{\prime}\right| \leq$ $c\left|\xi_{n}-\xi\right|$ for all $n \geq 1$ and $a+\delta \leq t \leq b-\delta$.

It was proved in [15] that for $f \in C^{1}$ in presence of a lower and upper functions $\alpha$ and $\beta$ such that $\alpha<\beta$ for $(a, b)$ a solution $x(t)$ exists for any $A \in[\alpha(a), \beta(a)], B \in$ $[\alpha(b), \beta(b)]$ such that the respective equation of variations $(7)$ is disconjugate on $(a, b)$.

Remark 8 (see [16, page 459]). It was mentioned by Erbe that under certain continuity conditions on the partial derivatives $f_{x}$, and $f_{x^{\prime}}$ the existence of a solution $x(t)$ of (1) with property
(B) implies that the corresponding equation of variations (7) along $x(t)$ is disconjugate on $(a, b)$; that is, the only solution of (7) with more than one zero on $I$ is the identically zero solution. Using the limiting process, we can say that the same is true for solutions $x(t)$ which possess the property $\left(B^{*}\right)$.

Also interesting for us is the following theorem, which Erbe has proved in [16].

Theorem 9 (see [16, Theorem 3.6, page 465]). Let $\alpha(t)$ be a lower solution and $\beta(t)$ an upper solution of (1) with $\alpha(t) \leq$ $\beta(t)$ on $[a, b]$ and $\alpha(a)<\beta(a), \alpha(b)<\beta(b)$. Assume that the Nagumo condition holds and let $g\left(x, x^{\prime}\right) \in G($ the class of all continuous functions $g\left(x, x^{\prime}\right)$ defined on $[\alpha(a), \beta(a)] \times R$ which are nondecreasing in $x^{\prime}$ and satisfy $g\left(\alpha(a), \alpha^{\prime}(a)\right) \geq$ $\left.0, g\left(\beta(a), \beta^{\prime}(a)\right) \leq 0\right)$ and $h\left(x, x^{\prime}\right) \in H($ the class of all continuous functions $h\left(x, x^{\prime}\right)$ defined on $[\alpha(b), \beta(b)] \times R$ which are nondecreasing in $x^{\prime}$ and satisfy $h\left(\alpha(b), \alpha^{\prime}(b)\right) \leq$ $\left.0, h\left(\beta(b), \beta^{\prime}(b)\right) \geq 0\right)$. Then there is a solution $x_{0}(t)$ of the BVP

$$
\begin{gathered}
x^{\prime \prime}=f\left(t, x, x^{\prime}\right), \\
g\left(x(a), x^{\prime}(a)\right)=0=h\left(x(b), x^{\prime}(b)\right)
\end{gathered}
$$

which satisfies $\alpha(t)<x_{0}(t)<\beta(t)$ on $[a, b]$.

Remark 10. If we select the function $g\left(x(a), x^{\prime}(a)\right)=-a_{1}$ $x(a)+a_{2} x^{\prime}(a)+A$ and $h\left(x(b), x^{\prime}(b)\right)=b_{1} x(b)+b_{2} x^{\prime}(b)-B$, then the inequalities (6) hold and the BVP (1), (5) has a solution on $[a, b]$.

Theorem 11 (see [16, Theorem 4.5, page 469]). Assume all hypotheses of Theorem 9 and, in addition, assume that the Lipschitz condition (there exist two nonnegative constants $K$ and $L$ such that whenever $\left(t, y, y^{\prime}\right)$ and $\left(t, x, x^{\prime}\right)$ are in the domain of definition of $f$, the inequality $\left|f\left(t, y, y^{\prime}\right)-f\left(t, x, x^{\prime}\right)\right| \leq$ $K|y-x|+L\left|y^{\prime}-x^{\prime}\right|$ holds) with respect to $x$ and $x^{\prime}$ holds. Then there is a solution $x_{0}(t)$ of the BVP

$$
\begin{gathered}
x^{\prime \prime}=f\left(t, x, x^{\prime}\right), \\
g\left(x(a), x^{\prime}(a)\right)=0=h\left(x(b), x^{\prime}(b)\right)
\end{gathered}
$$

which has property $\left(B^{*}\right)$ and satisfies $\alpha(t) \leq x_{0}(t) \leq \beta(t)$ on $[a, b]$.

\section{Solutions of Nonzero Type}

It follows from the above results by Knobloch-Jackson-Schrader-Erbe that in presence of lower and upper functions $\alpha$ and $\beta$ most two-point boundary value problems for (1) have solutions $\xi(t)$ with the property that the respective linear equation of variations

$$
y^{\prime \prime}=f_{x}\left(t, \xi(t), \xi^{\prime}(t)\right) y+f_{x^{\prime}}\left(t, \xi(t), \xi^{\prime}(t)\right) y^{\prime}
$$

is disconjugate in $(a, b)$. Generally properties of $y(t)$ can be made more precise for the Neumann problem, mixed type boundary conditions

$$
x(a)=A, \quad x^{\prime}(b)=B_{1}
$$


or

$$
x^{\prime}(a)=A_{1}, \quad x(b)=B
$$

or Sturm - Liouville type boundary conditions (5).

We restrict ourselves to the Dirichlet boundary conditions (3).

In presence of $\alpha$ and $\beta$ such that $\alpha<\beta$ for $(a, b)$ and provided a Nagumo type condition holds, a solution $x(t)$ of the problem (1), (3) exists which is such that the equation of variations is disconjugate in $(a, b)$. Therefore classical theorems on existence of a solution of the BVP (1), (3) can be treated as results on existence of a specific solution possessing properties which in different sources are denoted $(B),\left(B^{*}\right)$, or $\left(B^{\prime}\right)$.

Simple examples show however that there are multiple solutions which do not possess the above properties. Indeed, consider the problem

$$
x^{\prime \prime}=-k^{2} x+x^{3}, \quad x(0)=0, x(1)=0,
$$

where $k$ is a parameter. Evidently there is the trivial solution $\xi(t) \equiv 0$. The respective equation of variations is $y^{\prime \prime}=-k^{2} y$. If $k>2 \pi$, then $y(t)$ has zeros in $(0,1)$. There are the lower and upper functions $\alpha=-k^{2}-1$ and $\beta=k^{2}+1$. Therefore there exist also solutions which possess the property of disconjugacy in $(0,1)$ of the respective equations of variations. In fact there are multiple solutions of BVP (15).

We consider boundary value problem

$$
\begin{gathered}
x^{\prime \prime}=f\left(t, x, x^{\prime}\right) \\
x(a)=A, \quad x(b)=B .
\end{gathered}
$$

In what follows we shall assume that

$$
\begin{aligned}
\left(A_{1}\right) & f\left(t, x, x^{\prime}\right), f_{x}\left(t, x, x^{\prime}\right) \text {, and } f_{x^{\prime}}\left(t, x, x^{\prime}\right) \in C([a, b] \times \mathbb{R} \times \\
\mathbb{R} \rightarrow \mathbb{R}) ; &
\end{aligned}
$$

$\left(A_{2}\right)$ there exist the lower and upper functions $\alpha(t)$ and $\beta(t)$ for equation $x^{\prime \prime}=f\left(t, x, x^{\prime}\right)$ such that $\alpha(t) \leq \beta(t)$ on $[a, b]$;

$\left(A_{3}\right) \alpha(a) \leq A \leq \beta(a)$ and $\alpha(b) \leq B \leq \beta(b) ;$

$\left(A_{4}\right)$ the Nagumo condition is satisfied in $\omega(\alpha, \beta)$.

Theorem 12 (see [5, Theorem 7.34, page 327]). Suppose that conditions $\left(A_{1}\right)-\left(A_{4}\right)$ are satisfied. Then the boundary value problem (16) has a solution $x(t)$ satisfying

$$
\alpha(t) \leq x(t) \leq \beta(t) \text { on }[a, b] .
$$

Theorem 13. Suppose that conditions $\left(A_{1}\right)-\left(A_{4}\right)$ are satisfied. Let $\alpha<\beta$. If $A$ and $B$ are constants such that

$$
\alpha(a)<A<\beta(a), \quad \alpha(b)<B<\beta(b),
$$

then there exist sequences $\left\{\underline{x}_{n}\right\}$ and $\left\{\bar{x}_{n}\right\}$, such that

$$
\begin{aligned}
& \beta(t)>\bar{x}_{1}(t)>\bar{x}_{2}(t)>\cdots>\bar{x}_{n}(t)>\cdots x^{*}(t) \\
& \alpha(t)<\underline{x}_{1}(t)<\underline{x}_{2}(t)<\cdots<\underline{x}_{n}(t)<\cdots x_{*}(t),
\end{aligned}
$$

where $x^{*}(t)$ and $x^{*}(t)$ are solutions of problem (16) such that $x_{*}(t) \leq x^{*}(t)$ for $t \in[a, b]$.
Proof. We construct the sequence $\left\{\bar{x}_{n}(t)\right\}$. Choose $\bar{A}_{i} \in(A$, $\beta(a))$ such that $\bar{A}_{i}$ monotonically converge to $A$. Choose $\bar{B}_{i} \in$ $(B, \beta(b))$ such that $\bar{B}_{i}$ monotonically converge to $B$. Consider the problem

$$
x^{\prime \prime}=f\left(t, x, x^{\prime}\right), \quad x(a)=\bar{A}_{1}, x(b)=\bar{B}_{1} .
$$

The inequalities

$$
\alpha(a)<A<\bar{A}_{1}<\beta(a), \quad \alpha(b)<B<\bar{B}_{1}<\beta(b)
$$

hold. Then a solution $\bar{x}_{1}(t)$ exists, such that $\alpha(t)<\bar{x}_{1}(t)<$ $\beta(t)$. Set $\beta_{1}(t)=\bar{x}_{1}(t)$. Consider the problem

$$
x^{\prime \prime}=f\left(t, x, x^{\prime}\right), \quad x(a)=\bar{A}_{2}, x(b)=\bar{B}_{2} .
$$

This is solvable because $\alpha(t), \beta_{1}(t)=\bar{x}_{1}(t)$ exist and

$$
\alpha(a)<\bar{A}_{2}<\bar{A}_{1}=\beta_{1}(a), \quad \alpha(b)<\bar{B}_{2}<\bar{B}_{1}=\beta_{1}(b) .
$$

Then $\bar{x}_{2}(t)$ exists such that $\alpha(t)<\bar{x}_{2}(t)<\bar{x}_{1}(t)$. Proceeding this way, we construct $\left\{\bar{x}_{n}(t)\right\}$. Consider

$$
\alpha(t)<\cdots<\bar{x}_{n}(t)<\cdots<\bar{x}_{2}(t)<\bar{x}_{1}(t)<\beta(t) .
$$

Applying the Arzela - Ascoli criterium (([5, Theorem 8.26, page 347$]$ ) let $E$ be a compact subset of $\mathbb{R}^{m}$ and let $\left\{f_{k}\right\}$ be a sequence of $n$-dimensional vector functions that is uniformly bounded and equicontinuous on $E$. Then there is a subsequence $\left\{f_{k_{j}}\right\}$ that converges uniformly on $E$ ) we can show (Theorem 2 in ([17])) that $\left\{\bar{x}_{n}\right\}$ contains a subsequence which converges to $x^{*}$.

The same type arguments show that $\left\{\underline{x}_{n}\right\}$ also exists and subsequence of $\left\{\underline{x}_{n}\right\}$ converges to $x_{*}$. Notice that $\underline{x}_{n}<x^{*}$ by construction and, therefore, $x_{*} \leq x^{*}$.

Remark 14. Solutions $x_{*}(t)$ and $x^{*}(t)$ may coincide; that is, $x_{*}(t) \equiv x^{*}(t)$. For instance, there are the upper and lower functions $\beta(t)=1$ and $\alpha(t)=-1$ for the problem $x^{\prime \prime}=x$, $x(0)=x(1)=0$, which has only the trivial solution. Therefore $x_{*}(t)=x^{*}(t) \equiv 0$.

Consider a linear equation of variations constructed for a particular solution $\xi(t)$ of the BVP (16). We will say that $\xi(t)$ possesses the property $(*)$ if equation of variations (12) with the initial conditions

$$
y(a)=0, \quad y^{\prime}(a)=1
$$

has a solution $y(t)$ such that $y(t) \neq 0, \forall t \in(a, b)$. By Sturm separation theorem this is equivalent to the assertion that no nontrivial solution $y(t)$ of (12) has more than one zero in $(a, b)$. Recalling the terminology in the papers $[13,15,16]$, we may say that the equation of variations (12) is disconjugate in $(a, b)$.

By construction, solutions $x_{*}$ and $x^{*}$ possess the property $(*)$. Therefore the respective solutions $y(t)$ of the equations of variations, satisfying the conditions (25), do not vanish in $(a, b)$. 
As example (15) shows, there are solutions, which do not possess the property $(*)$. The respective $y(t)$ (the solutions of $(12),(25))$ are oscillatory functions, which may have multiple zeros.

We are led thus to the following classification of solutions of the problem (16).

Definition 15. Let $\xi(t)$ be a solution of the problem (16). One says that $\xi(t)$ is a zero type solution, if the equation of variations with respect to $\xi(t)$

$$
y^{\prime \prime}=f_{x}\left(t, \xi(t), \xi^{\prime}(t)\right) y+f_{x^{\prime}}\left(t, \xi(t), \xi^{\prime}(t)\right) y^{\prime}
$$

is such that a solution $y(t)$ with the initial conditions $y(a)=$ $0, y^{\prime}(a)=1$ does not have zeros in $(a, b)$ and $y(b) \neq 0$. Denote this type $(\xi)=0$.

If the above is valid and $y(b)=0$, one writes type $(\xi)=$

We extend definition of the type of a solution.

Definition 16. Let $\xi(t)$ be a solution of Dirichlet problem. One says that the type of $\xi(t)$ is $i(i \neq 0)$, if equation of variations with respect to $\xi(t)$ is such that a solution $y(t)$ with the initial conditions $y(a)=0, y^{\prime}(a)=1$ has exactly $i$ zeros in the interval $(a, b)$ and $y(b) \neq 0$. Denote this type $(\xi)=i$. If moreover $y(b)=0$, denote type $(\xi)=(i, i+1)$.

Remark 17. Therefore, a solution of type $(i-1, i)$ is a solution $\xi(t)$ such that the respective $y(t)$ has exactly $(i-1)$ zeros in $(a, b)$ and $y(b)=0$ also.

Remark 18. If $\xi(t)$ is an $i$-type solution of the problem (16) according to Definition 16, then for small enough $\gamma>0$ the difference

$$
u(t, \gamma)=x(t, \gamma)-\xi(t), \quad x(a, \gamma)=\xi(a)
$$

has exactly $i$ zeros in the interval $(a ; b)$ and $u(b, \gamma) \neq 0$. Solutions $x(t, \gamma)$ of the initial value problem $x^{\prime \prime}=f\left(t, x, x^{\prime}\right)$, $x(a)=A, x^{\prime}(a)=\gamma$ will be called neighboring solutions to solution $\xi(t)$.

In Theorem 13 approximating sequences are monotone. This is the reason why the limiting solutions $x^{*}$ and $x_{*}$ possess the property $(*)$. There may be also nonmonotone sequences also converging to some solutions of the BVP (16). Imagine that auxiliary boundary value problems $x(a)=$ $A_{n}, x(b)=B_{n}$ are defined for $A_{n}>A$, but $B_{n}<B$. We will call this type of sequences diagonal and sequences defined by the choice $A_{n}>A, B_{n}>B$ or $A_{n}<A, B_{n}<B$ straight sequences. The diagonal sequences also can be shown to satisfy the Arzela-Ascoli criterium. Therefore they also contain converging subsequences. If there is a unique solution in the problem (16) then both straight and diagonal sequences should converge to it. The diagonal sequence however cannot be monotone, by construction.

The following two results shed light on convergence of straight and diagonal sequences.

Theorem 19 (see [17, Theorem 3]). Let the conditions of Theorem 13 hold. If there exists a sequence $\left\{x_{n}\right\}$, consisting of solutions of the same type $i(i \neq 0)$ of the above auxiliary problems and $\alpha(t)<x_{n}(t)<\beta(t)$ in $[a, b]$, then there exists $a$ subsequence converging to a similar type solution $x(t)$ of the problem (16). Thus either type $(x)=(i-1, i)$, or type $(x)=i$, or type $(x)=(i, i+1)$.

Theorem 20. Let $\xi(t)$ be a solution of problem (16) and type( $\xi)$ $=i$. Then there exists a sequence $\left\{x_{n}\right\}$ such that type $\left(x_{n}\right)=i$, where $x_{n}$ are solutions of auxiliary problems

$$
\begin{array}{r}
x^{\prime \prime}=f\left(t, x, x^{\prime}\right), \quad x(a)=A_{n}, \quad x(b)=B_{n}, \\
\text { where } A_{n} \longrightarrow A, B_{n} \longrightarrow B .
\end{array}
$$

Proof. Let a solution $\xi(t)$ of the problem (16) have type $(\xi(t))=$ $i$. This means that a solution $y(t)$ of problem (12), (25) has exactly $i$ zeros in $(a, b)$ and $y(b) \neq 0$.

We wish to construct a sequence $x_{n}\left(t ; \varepsilon_{n}\right)$ of solutions of auxiliary problems

$$
\begin{aligned}
& x^{\prime \prime}=f\left(t, x, x^{\prime}\right), \quad x(a)=A_{n}, \\
& x(b)=B_{n}, \quad A_{n} \longrightarrow A, \quad B_{n} \longrightarrow B,
\end{aligned}
$$

which are of the same type $i$ and which converge to $\xi(t)$.

Consider solutions $x_{n}\left(t ; \varepsilon_{n}\right)$ of the problems

$$
\begin{array}{r}
x^{\prime \prime}=f\left(t, x, x^{\prime}\right), \quad x(a)=\xi(a)=A, \\
x^{\prime}(a)=\xi^{\prime}(a)+\varepsilon_{n} .
\end{array}
$$

If $\varepsilon_{n} \rightarrow 0$, then $x_{n}\left(b ; \varepsilon_{n}\right) \rightarrow \xi(t)$. Moreover, the functions $y_{n}\left(t ; \varepsilon_{n}\right)$ which are solutions of the Cauchy problems

$$
\begin{array}{r}
y^{\prime \prime}=f_{x}\left(t, x_{n}, x_{n}^{\prime}(t)\right) y+f_{x^{\prime}}\left(t, x_{n}(t), x_{n}^{\prime}(t)\right) y^{\prime}, \\
y(a)=0, \quad y^{\prime}(a)=1
\end{array}
$$

are similar to $y(t)$ and hence have exactly $i$ zeros in $(a, b)$, $y_{n}\left(b, \varepsilon_{n}\right) \neq 0$. Then $x_{n}\left(t ; \varepsilon_{n}\right)$ have type $i$. Take finally, $A_{n}=A$, $B_{n}=x_{n}\left(b ; \varepsilon_{n}\right)$, and the required sequence is constructed.

Let us summarize the results of this section.

In presence of a regular pair $\alpha$ and $\beta$ of lower and upper solutions and under the Nagumo condition a solution of the Dirichlet boundary value problem (16) exists. Always there is a solution of type $(0)$ or type $(0,1)$. These solutions can be approximated by a monotone sequence of solutions of auxiliary boundary value problems.

Solutions of nonzero type cannot be approximated by monotone sequences. They can be approximated by suitable straight or diagonal sequences. It follows that straight and diagonal sequences of solutions can be constructed.

The auxiliary boundary value problems (which contain elements of straight or diagonal sequences) can have multiple solutions. These solutions can be arranged in sequences of similar type solutions. These sequences contain subsequences converging (accordingly to Arzela - Ascoli criterium) to solutions of different type of a given boundary value problem.

An $i$-type solution of the problem (16) can be approximated by similar type solutions of auxiliary boundary value problems. 


\section{Neumann Problem}

The Neumann problem

$$
x^{\prime \prime}=f\left(t, x, x^{\prime}\right), \quad x^{\prime}(a)=A_{1}, x^{\prime}(b)=B_{1}
$$

can be considered as a sample of problems with Sturm Liouville boundary conditions. This type of problems is more difficult to treat through types of solutions since it is not so easy to define the types. For the Neumann boundary conditions this difficulty appears as nonstability of zeros of the difference $x^{\prime}(t ; \gamma)-\xi^{\prime}(t)$ under the change of $\gamma, x(t ; \gamma)$ being a solution of the initial value problem $x^{\prime \prime}=f\left(t, x, x^{\prime}\right)$, $x(a)=\gamma, x^{\prime}(a)=A_{1}$ and $\xi(t)$ being a solution of the problem (32). For instance, considering solutions $x(t ; \gamma)$ of the initial problem $x^{\prime \prime}=f\left(t, x, x^{\prime}\right), x(a)=A, x^{\prime}(a)=\gamma$, one can employ the fact that zeros of $x(t ; \gamma)-\xi(t)$, where $\xi$ is a solution of the BVP, are stable with respect to small changes of $\gamma$. This is not the case for zeros of $x^{\prime}(t ; \gamma)-\xi^{\prime}(t)$. One can overcome this difficulty by special introduction of types of solutions. This was done in [18].

\section{Types of Solutions and Multiplicity}

It appears that the existence of a solution of nonzero type indicates that there are additional solutions.

Consider the Dirichlet problem (16).

Theorem 21. Suppose that all solutions of equation $x^{\prime \prime}=f(t$, $\left.x, x^{\prime}\right)$ are extendable to the interval $[a, b]$. If there are two solutions $u$ and $v$ of the problem (16), type $(u)=m$, type $(v)=n$, $|m-n| \geq 2$, then there exist at least $|m-n|-1$ more solutions of the problem (16).

Proof. We prove the result for the specific case $n=0$ and $m=2$. Our goal is to show that there exists at least one more solution of the problem. The proof for the general case can be conducted similarly.

Suppose that

$$
x_{n}^{\prime}(a)>x_{m}^{\prime}(a)
$$

Denote

$$
x_{n}^{\prime}(a)=p, \quad x_{m}^{\prime}(a)=q \quad(p>q) .
$$

Consider solutions $x(t ; \gamma)$ of the Cauchy problems

$$
x(a)=A, \quad x^{\prime}(a)=\gamma \in[q, p] .
$$

Obviously $x_{n}(t)=x(t, p), x_{m}(t)=x(t, q)$.

Introduce function

$$
u_{n}(t, \gamma)=x_{n}(t)-x(t, \gamma) .
$$

If $\gamma<p$ and $\gamma$ is close enough to $p$, then $u_{n}(t ; \gamma)$ has not zeros in $(a, b)$. Several cases are possible.

Case 1. $u_{n}(t, \gamma) \neq 0$ in $(a, b)$ for all $\gamma \in(q, p)$.

Then $u_{n}(t ; q) \neq 0$ in $(a, b)$. This means that $x_{n}$ and $x_{m}$ do not intersect in $(a, b)$, but

$$
x_{n}(b)=x_{m}(b) .
$$

Then consider function

$$
u_{m}(t, \gamma)=x(t, \gamma)-x_{m}(t)
$$

Function $u_{m}(t ; \gamma)$ has two zeros $t_{1}(\gamma)$ and $t_{2}(\gamma)$ in $(a, b)$ and

$$
u_{m}(b ; \gamma) \neq 0
$$

for $\gamma>q$ and close enough to $q$. One has that

$$
u_{m}(t, p)=x(t, p)-x_{m}(t)=x_{n}(t)-x_{m}(t)=u_{n}(t, q)
$$

and $u_{m}(t, p)$ has not zeros in $(a, b)$. It follows that

$$
t_{2}(\gamma)=b, \quad t_{1}(\gamma)=b
$$

for some $\gamma_{2}<\gamma_{1} \leq p$.

A solution $x\left(t, \gamma_{2}\right) \not \equiv x_{m}(t)$ (and $\left.x\left(t, \gamma_{2}\right) \not \equiv x_{n}(t)\right)$ solves the BVP (16).

Case 2. Function $u_{n}(t, \gamma)$ has not zeros in $(a, b]$ for $\gamma<p$ and $\gamma$ close enough to $p$, and

$$
u_{n}(t, q)=x_{n}(t)-x_{m}(t)
$$

has a zero at $t=b$. Let $\gamma_{1} \in[q, p)$ be such that $u_{n}(t, \gamma)$ has a zero at $t=b$ for the first time.

If $\gamma_{1}=q$, then Case 2 reduces to Case 1 .

If $\gamma_{1} \in(q, p)$, then $x\left(t, \gamma_{1}\right)$ is a solution of BVP (16) and $x\left(t, \gamma_{1}\right)$ is different from $x_{n}$ and $x_{m}$. Hence the proof.

Theorem 22. Suppose that there exist lower and upper functions $\alpha$ and $\beta$ in the problem (16) and the Nagumo condition holds. Suppose also that there exists a solution $\xi(t)$ of the type $k(k>1)$, and $\xi(t)$ is located between $\alpha(t)$ and $\beta(t)$. Then there exist at least $2 k$ other solutions.

Proof. Since $\xi(t)$ is located between $\alpha(t)$ and $\beta(t)$ it does not coincide neither with $\beta$ nor with $\alpha$. Consider the region $\omega(\xi, \beta)$ between $\xi$ and $\beta$. One may consider $\xi$ as a lower function for this region since all of the conditions for lower functions are fulfilled. Then there exists a solution $x_{0}(t)$ of zero type in this region. Consider now solutions $\xi$ and $x_{0}$ in the region $\omega(\alpha, \beta)$. By Theorem 21 , there exist more $k-1$ solutions of the problem. Totally there are $k$ solutions (with $x_{0}$ ) not counted as a solution $\xi$.

The same analysis, made for the region $\omega(\alpha, \xi)$, shows that there are at least $k$ solutions in this region.

Then the total number of solutions of the BVP in the region $\omega(\alpha, \beta)$ is at least $2 k$, a solution $\xi$ not counted.

\section{Quasilinearization and Multiple Solutions of BVP}

Consider the problem

$$
\begin{aligned}
& \left(l_{2} x\right)(t)=f\left(t, x, x^{\prime}\right), \\
& x(a)=0, \quad x(b)=0,
\end{aligned}
$$


where $\left(l_{2} x\right)(t)=x^{\prime \prime}+p(t) x^{\prime}+q(t) x$ is the second order linear differential expression with continuous coefficients. It is well known ([19] and similar results in $[1,2,4])$ that the above problem is solvable if $f$ is bounded $\left(\left|f\left(t, x, x^{\prime}\right)\right|<M\right.$ for all $\left.\left(t, x, x^{\prime}\right)\right)$ and the homogeneous problem

$$
\left(l_{2} x\right)(t)=0, \quad x(a)=0, x(b)=0
$$

has only the trivial solution.

Introduce the following notions $([20,21])$.

Definition 23. One will say that the linear part $\left(l_{2} x\right)(t)$ is $i$ nonresonant with respect to the boundary conditions (44), if a solution $x(t)$ of the Cauchy problem

$$
\left(l_{2} x\right)(t)=0, \quad x(a)=0, \quad x^{\prime}(a)=1
$$

has exactly $i$ zeros in the interval $(a, b)$ and $x(b) \neq 0$.

Definition 24. One will say that $\xi(t)$ is an $i$-type solution of the problem (43), (44), if for small enough $r>0$ the difference $u(t ; r)=x(t ; r)-\xi(t)$ has exactly $i$ zeros in $(a, b)$ and $u(b ; r) \neq$ 0 , where $x(t ; r)$ is a solution of (43), which satisfies the initial conditions

$$
x(a ; r)=\xi(a), \quad x^{\prime}(a ; r)=\xi^{\prime}(a) \pm r .
$$

Remark 25. In terms of the previous section the type of $\xi(t)$ is either $i$ or $(i, i+1)$.

The following result is crucial.

Theorem 26. Quasilinear problem (43), (44) with an i-nonresonant linear part $\left(l_{2} x\right)(t)$ has an i-type solution.

The proof can be found in $[20,21]$. It is based on a series of lemmas. First a set of all solutions $S$ of the problem (43), (44) is considered. It is not empty, by Conti's theorem [19], and it is $C^{1}$-compact, by using the Green's function representation of a solution. Moreover, any possible solution $x(t)$ of the problem satisfies the estimate

$$
\begin{aligned}
& |x(t)| \leq \Gamma \cdot M(b-a), \\
& \left|x^{\prime}(t)\right| \leq \Gamma_{1} \cdot M(b-a) \quad \forall t \in[a, b],
\end{aligned}
$$

where $M$ is a bound for $f, \Gamma$ and $\Gamma_{1}$ are estimates of the respective Green's function and its derivative in $t$. There are elements in $S$ with maximal $x^{\prime}(a)$ and minimal $x^{\prime}(a)$ (they coincide in case of a unique solution of the problem). These solutions possess the needed property of being of type $i$. This can be concluded by consideration of solutions of the Cauchy problem (43), $x(a)=0, x^{\prime}(a)=\gamma$ and letting $\gamma$ to go to $\pm \infty$. The normalized functions $x(t ; \gamma) / \gamma$ go (as $\gamma$ goes to $\pm \infty$ ) to solutions of the Cauchy problem $\left(l_{2} z\right)(t)=0, z(a)=0$, $z^{\prime}(a)= \pm 1$, which have exactly $i$-zeros in $(a, b)$. Thus in case the maximal and minimal solutions are not of $i$-type, they cannot possess, respectively, the maximality and minimality properties.

Theorem 26 can be used to detect multiple solutions of boundary value problems. Indeed, suppose that the problem

$$
x^{\prime \prime}=f(t, x), \quad x(a)=0, x(b)=0
$$

is considered. Rewrite the equation in equivalent form

$$
x^{\prime \prime}+k^{2} x=f(t, x)+k^{2} x
$$

and truncate the right side by a constant $N>0$. Denote the truncated (thus bounded) continuous right side $F_{N}(t, x)$. Let $M$ be the estimate for $\left|F_{N}(t, x)\right|$. Let $\Gamma$ be the estimate for the Green's function $G(t, x)$ of the problem $x^{\prime \prime}+k^{2} x=0, x(a)=$ $0, x(b)=0$. Evidently, $|x(t)| \leq \Gamma \cdot M(b-a)$, where $x$ is a solution of the modified problem

$$
x^{\prime \prime}+k^{2} x=F_{N}(x), \quad x(a)=0, x(b)=0 .
$$

Then, if the key inequality

$$
\Gamma \cdot M(b-a) \leq N
$$

holds, a solution $x(t)$ of the problem (51) solves also the problem (50). It is important that the type of $x(t)$ is the same as the type of a linear part $x^{\prime \prime}+k^{2} x$.

Repeating this quasilinearization process multiplying with linear parts of different types, we obtain multiple solutions of the problem (50). All of these solutions are different since they are of different types.

The applicability of the quasilinearization process was demonstrated in the papers [20-24].

\section{Resonant Problems}

The above mentioned Conti's theorem [19] is not applicable for boundary value problems (43), (44) if the respective homogeneous problem (45) has a nontrivial solution. This is the so-called resonant case. There are numerous papers devoted to resonant boundary value problems. Some of them can be found in the bibliography of papers [25-27].

The classical result says that the simple self-adjoint resonant problem

$$
x^{\prime \prime}+p x^{\prime}+q x=F(t), \quad x(a)=0, x(b)=0
$$

is solvable if $F$ is "orthogonal" to eigenfunction of a linear part. In a more general setting

$$
\begin{gathered}
x^{\prime \prime}+p x^{\prime}+q x=F\left(t, x, x^{\prime}\right) \\
x(a)=0, \quad x(b)=0 .
\end{gathered}
$$

One should "measure" how "far" is F of being "orthogonal" to an eigenfunction of a linear part.

It seems that quasilinearization process described in the previous section is applicable to resonant BVPs also. The main idea is to modify a linear part and, respectively, the right side of an equation so that the "new" linear part is no more resonant with respect to the boundary conditions. Then the "new" right side is truncated by a constant $N$ and if the inequality (52) holds then the problem has a solution.

This idea was realized first in the work by Yermachenko [25]. The Dirichlet boundary conditions were considered. Later the results of the same spirit were obtained in the works $[26,27]$. In the work [26] the mixed boundary conditions 
$x^{\prime}(0)=0, x(T)=0$ were considered. The types of a linear part and of solutions were defined.

Below a sample of the results of this kind is given.

Consider the resonant boundary value problem

$$
\begin{gathered}
x^{\prime \prime}+p x^{\prime}+q x=F\left(t, x, x^{\prime}\right) \\
x(0)=0, \quad x^{\prime}(T)=0 .
\end{gathered}
$$

Theorem 27. Supposing for the modified BVP

$$
\begin{gathered}
x^{\prime \prime}+p x^{\prime}+q x+\varepsilon^{2} x=\varphi\left(t, x, x^{\prime}\right), \\
x(0)=0, \quad x^{\prime}(T)=0,
\end{gathered}
$$

where

(1) $\varphi\left(t, x, x^{\prime}\right)=\varepsilon^{2} \delta(-N, x, N)+F(t, \delta(-N, x, N)$, $\left.\delta\left(-N_{1}, x^{\prime}, N_{1}\right)\right), \delta$ is the truncation function, $N>0$, $N_{1}>0$ and

(2) linear part is no more resonant with respect to the given boundary conditions (56),

the inequalities

$$
\begin{gathered}
\Gamma \cdot M \cdot T \leq N, \\
\Gamma_{1} \cdot M \cdot T \leq N_{1}
\end{gathered}
$$

hold, where $\Gamma, \Gamma_{1}$ are the estimates of the Green's function and its derivative associated with the linear part in (57) and $M=$ $\sup _{I \times R^{2}}\left|\varphi\left(t, x, x^{\prime}\right)\right|$.

Then the problem (55), (56) has a solution such that $|x(t)| \leq$ $N \forall t \in[a, b]$ and $\left|x^{\prime}(t)\right| \leq N_{1} \forall t \in[a, b]$.

Proof. Consider the modified problem (57). It is solvable, since the linear part is not more resonant and the nonlinearity in the right side is bounded. A solution $x(t)$ of (57) can be written in the integral form using the Green's function.

The inequalities (58), (59) fulfill. This means that

$$
|x(t)| \leq N, \quad\left|x^{\prime}(t)\right| \leq N_{1}, \quad \forall t \in[a, b] .
$$

For these values of $x$ and $x^{\prime}$ the original equation (55) and the modified equation (57) are equivalent.

It follows that $x(t)$ is also a solution of the original problem (55), (56).

Remark 28. If the right side in (55) does not depend on $x^{\prime}$, then $N_{1}$ can be set to $+\infty$ and therefore only the inequality (58) should be verified.

\section{Conclusions}

General results like Conti's theorem provide information on the existence of a solution of BVP. Applying these results to specific problems like the Dirichlet problem for the second order ordinary differential equations one should obtain some estimates of expected solutions and verify a set of conditions.

The classical example is the method of upper and lower functions. If the regularly ordered $(\alpha \leq \beta)$ upper and lower functions exist and satisfy some restrictions depending on boundary conditions then the existence of a solution is established. For equations depending on the derivative of solution additional conditions of the Nagumo type may be needed. It was observed that in presence of regular upper and lower functions a solution of the BVP exists which possesses the specific property; namely, the respective equation of variations is disconjugate (nonoscillatory) in a given interval. These solutions can be approximated by monotone sequences of solutions of auxiliary problems.

It seems to be useful to classify solutions of BVP by oscillatory behaviour of their equations of variations. The above described solutions are referred to as zero type solutions. Solutions are classified, roughly speaking, with respect to the number of zeros of the respective equation of variations in a given interval. Solutions of nonzero type cannot be approximated by monotone sequences. They can be approximated however by nonmonotone sequences of solutions of auxiliary problems.

In case of the existence of regular upper and lower functions the existence of a solution of nonzero type implies the existence of additional solutions of BVP. If there are no zero type solutions of BVP, the method of upper and lower functions is not applicable to the problem (there are no upper and lower functions which are not solutions of BVP).

Even in case of nonexistence of upper and lower functions a priori information on the type of possible solutions is useful and may yield practical estimations of the number of solutions.

A priori knowledge of the type of a solution of BVP allows applying suitable quasilinearization process and confirming the existence of a solution.

Resonant problems which have a solution can be handled also. If the quasilinearization process is applicable then the solvability of a solution is proved and the type of a solution can be detected.

Finally, knowledge of the type of a solution makes it easier to construct the approximating sequences.

\section{Conflict of Interests}

The authors declare that there is no conflict of interests regarding the publication of this paper.

\section{Acknowledgments}

This work is supported partially by ESF project no. 2013/ 0024/1DP/1.1.1.2.0/13/APIA/VIAA/045 and Latvian Council of Science Grant no. 345/2012.

\section{References}

[1] S. R. Bernfeld and V. Lakshmikantham, An Introduction to Nonlinear Boundary Value Problems, Academic Press, New York, NY, USA, 1974.

[2] Yu. A. Klokov and N. I. Vasilyev, Foundations of the Theory of Nonlinear Boundary Value Problems, Zinatne, Riga, Latvia, 1978 (Russian).

[3] I. T. Kiguradze, Singular Boundary Value Problems for Ordinary Differential Equations, Tbilissi St. Univ. Press, Tbilissi, Georgia, 1975, (Russian). 
[4] R. P. Agarwal and D. O'Regan, An Introduction to Ordinary Differential Equations, Universitext, Springer, New York, NY, USA, 2008.

[5] W. G. Kelley and A. C. Peterson, The Theory of Differential Equations: Classical and Qualitative, Pearson Education, New Jersey, NJ, USA, 2004.

[6] P. Amster, Topological Methods in the St udy of Boundary Value Problems, Springer, 2014.

[7] L. H. Erbe, "A uniqueness theorem for second order differential equations," Mathematische Zeitschrift, vol. 109, no. 2, pp. 92-96, 1969.

[8] C. de Coster and P. Habets, Two-Point Boundary Value Problems: Lower and Upper Solutions, Elsevier, Amsterdam, The Netherlands, 2006.

[9] L. A. Lepin, "On the concepts of lower and upper functions," Differential Equations, vol. 16, no. 10, pp. 1750-1759, 1980 (Russian).

[10] L. A. Lepin, "Generalizaded solutions and the solvability of boundary value problems for a second order differential equation," Differential Equations, vol. 18, no. 8, pp. 925-931, 1982 (Russian).

[11] H. Epheser, "Über die existenz der lösungen von randwertaufgaben mit gewöhnlichen, nichtlinearen differentialgleichungen zweiter ordnung," Mathematische Zeitschrift, vol. 61, pp. 435454, 1955.

[12] F. Sadyrbaev, "Ljapunov functions and the solvability of the first boundary value problem for an ordinary second-order differential equation," Differentsial'nye Uravneniya, vol. 16, no. 4, pp. 629-634, 1980 (Russian).

[13] H. W. Knobloch, "Comparison theorems for nonlinear second order differential equations," Journal of Differential Equations, vol. 1, pp. 1-26, 1965.

[14] H. Knobloch, "Second order differential equalities and a nonlinear boundary value problem," Journal of Differential Equations, vol. 5, pp. 55-71, 1969.

[15] L. K. Jackson and K. W. Schrader, "Comparison theorems for nonlinear differential equations," Journal of Differential Equations, vol. 3, pp. 248-255, 1967.

[16] L. H. Erbe, "Nonlinear boundary value problems for second order differential equations," Journal of Differential Equations, vol. 7, pp. 459-472, 1970.

[17] M. Dobkevich, "Non-monotone convergence schemes," Mathematical Modelling and Analysis, vol. 17, no. 4, pp. 589-597, 2012.

[18] M. Dobkevich and F. Sadyrbaev, "Approximation schemes and types of solutions for the Neumann BVP," Proceedings of IMCS of University of Latvia, vol. 13, pp. 12-23, 2013.

[19] R. Conti, "Equazioni differenziali ordinarie quasilineari con condizioni lineari," Annali di Matematica Pura ed Applicata, vol. 57, pp. 49-61, 1962.

[20] I. Yermachenko and F. Sadyrbaev, "Types of solutions and multiplicity results for two-point nonlinear boundary value problems," Nonlinear Analysis, vol. 63, pp. e1725-e1735, 2005.

[21] I. Yermachenko and F. Sadyrbaev, "Quasilinearization and multiple solutions of the Emden-Fowler type equation," Mathematical Modelling and Analysis, vol. 10, no. 1, pp. 41-50, 2005.

[22] I. Yermachenko and F. Sadyrbaev, "Types of solutions of the second order Neumann problem: multiple solutions," Proceedings of IMCS of University of Latvia, vol. 4, pp. 5-21, 2004, http://www.lumii.lv/Pages/sbornik1/neumann.pdf.
[23] I. Yermachenko and F. Sadyrbaev, "Types of solutions and multiplicity results for second order nonlinear boundary value problems. Discrete and continuous dynamical systems," in Proceedings of AIMS Conference, vol. 2007, pp. 1061-1069, Poitier, France, 2006.

[24] I. Yermachenko and F. Sadyrbaev, "Quasilinearization technique for $\Phi$-Laplacian type equations," International Journal of Mathematics and Mathematical Sciences, vol. 2012, Article ID 975760, 11 pages, 2012.

[25] I. Yermachenko, "Two-point boundary value problems at resonance," Mathematical Modelling and Analysis, vol. 14, no. 2, pp. 247-257, 2009.

[26] N. Sveikate and F. Sadyrbaev, "Quasilinearization for resonant boundary value problems with mixed boundary conditions," Nonlinear Oscillations, vol. 17, no. 1, pp. 112-126, 2014.

[27] N. Sveikate, "Resonant problems by quasilinearization," International Journal of Differential Equations, vol. 2014, Article ID 564914, 8 pages, 2014. 


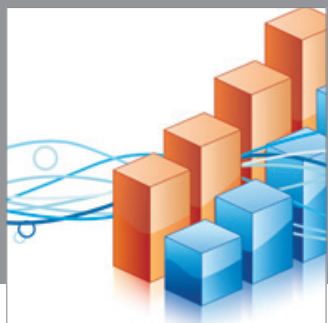

Advances in

Operations Research

mansans

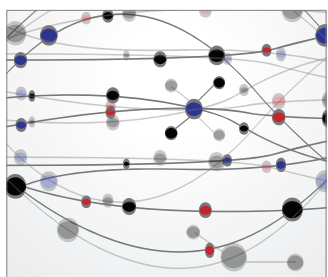

The Scientific World Journal
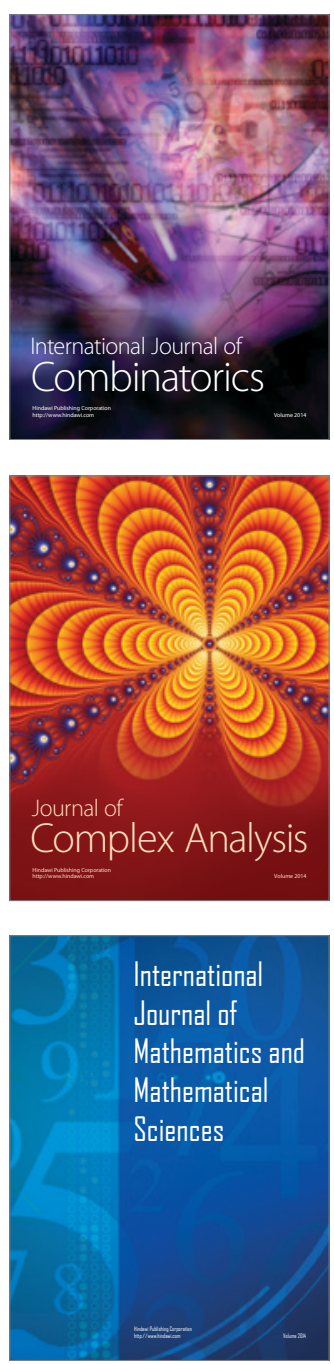
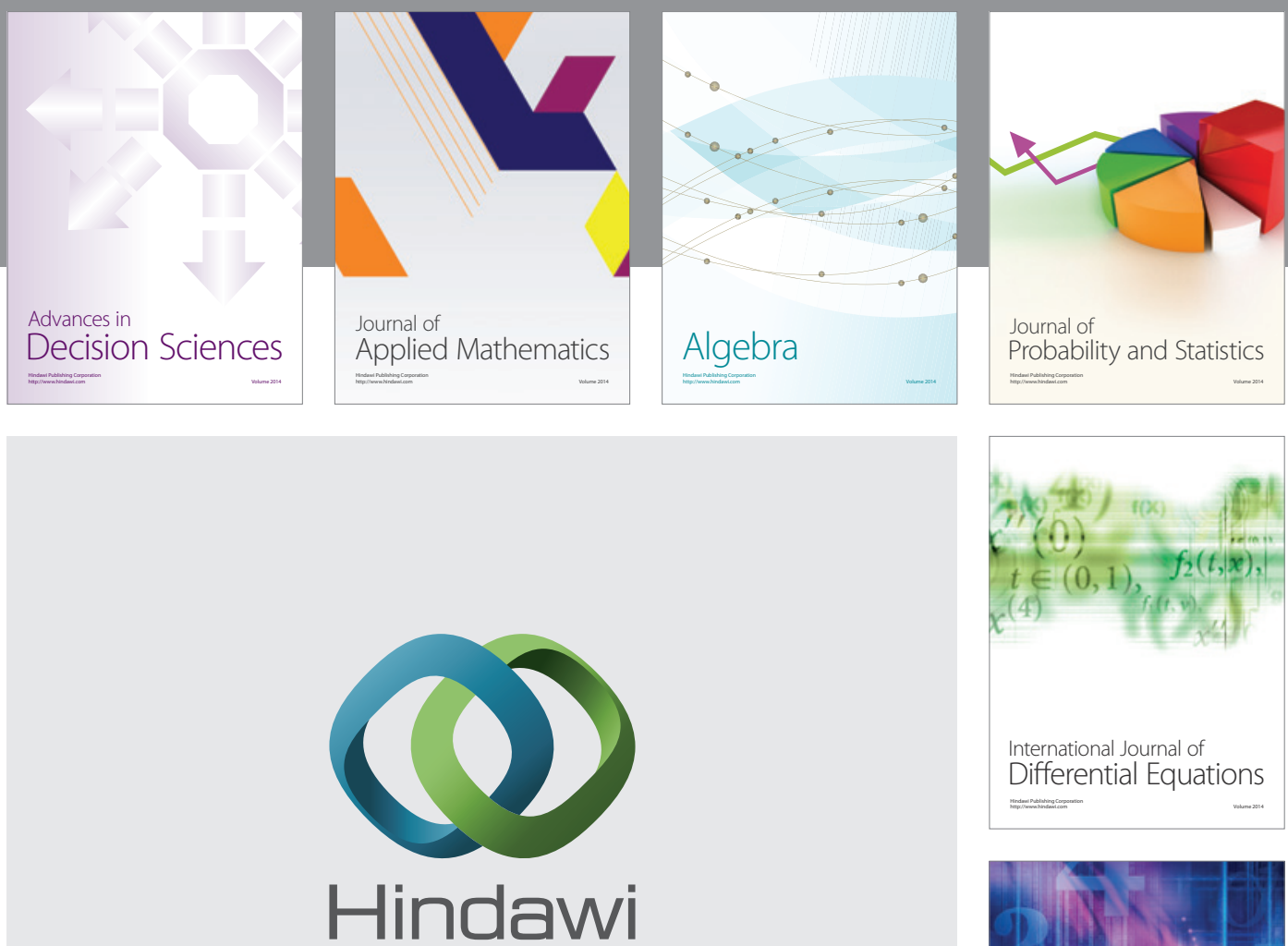

Submit your manuscripts at http://www.hindawi.com
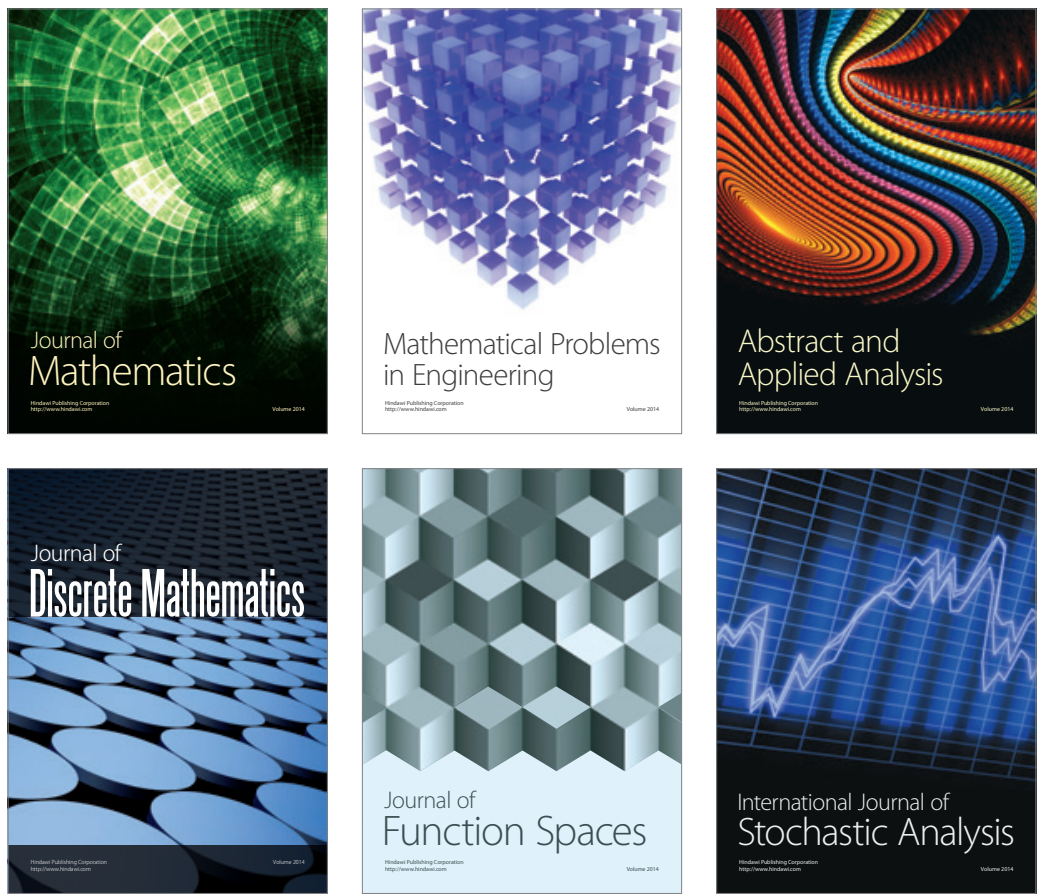

Journal of

Function Spaces

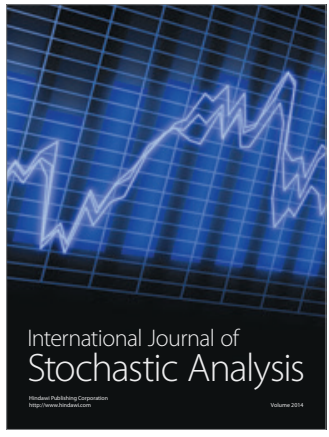

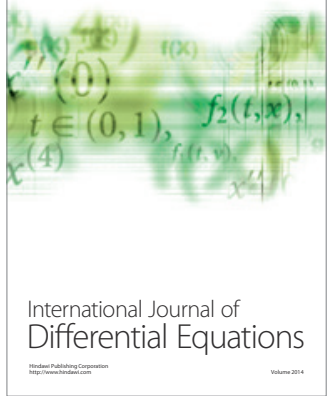
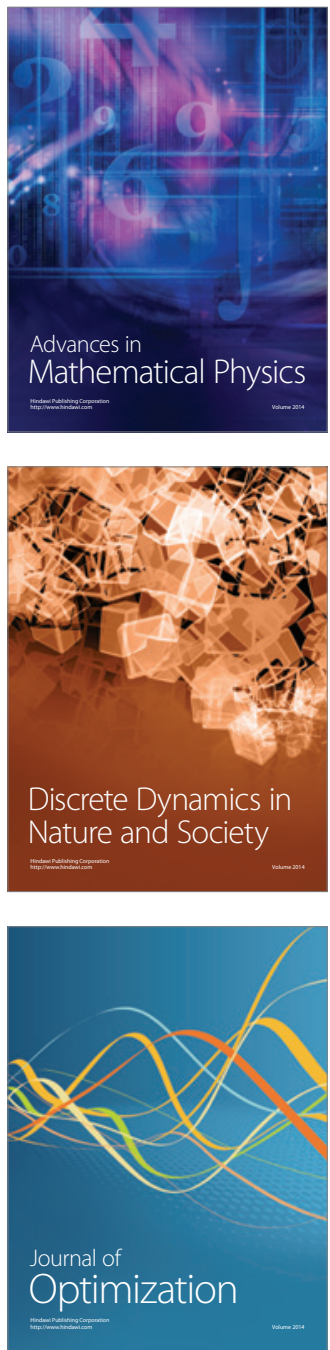